\title{
Reflets
}

Revue d'intervention sociale et communautaire

\section{L'intégration à l'emploi des personnes présentant une déficience intellectuelle et en situation linguistique minoritaire : état des lieux et pratiques prometteuses}

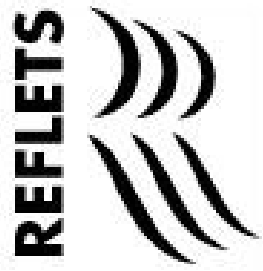

\section{Lynne A. Potvin, Natasha Plourde, Linda Cardinal et Virginie Cobigo}

Volume 24, numéro 1, printemps 2018

Les inégalités sociales en contexte minoritaire

URI : https://id.erudit.org/iderudit/1051520ar

DOI : https://doi.org/10.7202/1051520ar

Aller au sommaire du numéro

Éditeur(s)

Reflets, Revue d'intervention sociale et communautaire

ISSN

1203-4576 (imprimé)

1712-8498 (numérique)

Découvrir la revue

Citer cet article

Potvin, L. A., Plourde, N., Cardinal, L. \& Cobigo, V. (2018). L’intégration à l'emploi des personnes présentant une déficience intellectuelle et en situation linguistique minoritaire : état des lieux et pratiques prometteuses. Reflets,

24(1), 42-67. https://doi.org/10.7202/1051520ar
Résumé de l'article

L'article porte sur le thème de l'inclusion sociale des personnes présentant une déficience intellectuelle (DI) dans le domaine de l'intégration à l'emploi. Il accorde aussi une attention particulière à la situation des francophones présentant une DI, une situation mal connue dans le milieu de la recherche sur les handicaps. Le texte présente les résultats d'une recension des écrits scientifiques sur la formation professionnelle et l'intégration à l'emploi des personnes présentant une DI. Les résultats de cette recherche permettent de poser des jalons en vue de mieux comprendre la question des inégalités sociales en milieu francophone chez les personnes présentant une DI et de réfléchir aux approches les plus appropriées pour favoriser leur intégration à un emploi au sein de leur milieu.
Tous droits réservés @ $\mathbb{0}$ Reflets, Revue d’intervention sociale et communautaire, 2018 ce document est protégé par la loi sur le droit d'auteur. L'utilisation des services d'Érudit (y compris la reproduction) est assujettie à sa politique d'utilisation que vous pouvez consulter en ligne. 


\title{
L'intégration à l'emploi des personnes présentant une déficience intellectuelle et en situation linguistique minoritaire : état des lieux et pratiques prometteuses
}

\author{
Lynne A. Potvin \\ Doctorante, École de psychologie, Université d'Ottawa
}

Natasha Plourde

Doctorante, École de psychologie, Université d'Ottawa

Linda Cardinal, Ph. D.

École d'études politiques, Université d'Ottawa

Virginie Cobigo, Ph. D.

École de psychologie, Université d'Ottawa

\section{Remerciements}

Nous remercions le Conseil de recherches en sciences humaines du Canada (CRSH), qui finance nos activités dans le cadre du programme Fonds d'innovation sociale destiné aux communautés et aux collèges. Le projet de recherche a été réalisé en collaboration avec les responsables et les étudiants inscrits au sein de deux programmes : Interventions auprès des personnes ayant un handicap (IPH) et Intégration communautaire par l'éducation coopérative (ICEC). Nous remercions le Groupe de recherche sur l'inclusion sociale en Ontario (GRISO) - un groupe de recherche-collaborative logé au collège d'arts appliqués et de technologie, La Cité, à Ottawa, et à l'Association pour l'intégration sociale d'Ottawa (AISO) - de leurs appuis à ses travaux. Les auteures remercient également Patrick Labelle, bibliothécaire à l'Université d'Ottawa, de sa collaboration à la stratégie de recherche.

\section{Résumé}

L'article porte sur le thème de l'inclusion sociale des personnes présentant une déficience intellectuelle (DI) dans le domaine de l'intégration à l'emploi. Il accorde aussi une attention particulière à la situation des francophones présentant une DI, une situation mal connue dans le milieu de la recherche sur les handicaps. Le texte présente les résultats 
d'une recension des écrits scientifiques sur la formation professionnelle et l'intégration à l'emploi des personnes présentant une DI. Les résultats de cette recherche permettent de poser des jalons en vue de mieux comprendre la question des inégalités sociales en milieu francophone chez les personnes présentant une DI et de réfléchir aux approches les plus appropriées pour favoriser leur intégration à un emploi au sein de leur milieu.

Mots clés : déficience intellectuelle, inclusion sociale, inégalités sociales, mentorat, formation professionnelle, francophonie, minorité linguistique, formation postsecondaire

\section{Abstract}

Research demonstrates that the social inclusion of individuals presenting with intellectual disabilities (ID) is often limited due to disparities in obtaining meaningful employment. However, very little research addresses this important topic, and especially in the context of linguistic minority. This article focuses on the social inclusion of Francophones in Ontario presenting with ID, specifically in the area of employment integration, and aims to contribute to the limited body of literature addressing this area. It reviews the scientific literature on the vocational training and employment integration of persons with ID. From those results we can begin understanding that Francophones presenting with ID in Ontario experience various forms of social inequalities related to their disability; this gap is widened when they must claim supports and services in French. In conclusion, this article helps identify the existing social inequalities amongst this population and address the most appropriate approaches to support their social inclusion in the context of employment integration.

Key words: intellectual disability, social inclusion, social inequalities, mentoring, vocational training, francophonie, linguistic minority, postsecondary training

\section{Introduction}

De nature exploratoire, l'article présente les résultats d'une recension des écrits sur le thème de l'intégration à l'emploi des personnes présentant une déficience intellectuelle (DI). La recension a été réalisée en 2015-2016, en appui à un projet de recherche appliquée sur les pratiques prometteuses d'intégration à l'emploi de jeunes francophones de l'Ontario présentant une DI, inscrits à un programme à leur intention à la Cité, le collègue 
d'arts appliqués et de technologies d'Ottawa. Le projet avait pour objectifs d'identifier ces pratiques prometteuses dans les écrits scientifiques et d'en tirer des leçons pour bonifier l'offre de stages en emploi aux étudiants présentant une DI de la Cité. Une des

"La recherche sur la question de la DI et tout autre type de handicaps en milieu minoritaire francophone au Canada en est à ses balbutiements. " retombées du projet est qu'il a aussi servi à mettre en place un nouvel espace de recherche et de dialogue sur la DI en milieu franco-ontarien. La recherche sur la question de la DI et tout autre type de handicaps en milieu minoritaire francophone au Canada en est à ses balbutiements. Les travaux publiés sur le sujet sont récents (Bélanger, Paré et Gagné, 2017; Lirette, et collab., 2015; Bélanger, et collab. 2015; Bélanger et Duchesne, 2010) et portent de façon particulière sur les pratiques d'inclusion dans les écoles de langue française en Ontario et au Manitoba. Pour leur part, Lirette, et collab. (2015) ont étudié la perception du handicap visuel en milieu de travail au Nouveau-Brunswick.

La recension des écrits sur l'intégration à l'emploi, dont nous présentons les résultats ci-dessous, s'ajoute à ces quelques travaux. Cette recension présente les pratiques prometteuses dans le domaine de la formation professionnelle et l'intégration à l'emploi. Elle veut servir de levier au développement de la recherche en français en Ontario sur les personnes présentant une DI, mais son intention initiale est aussi de contribuer à l'amélioration des pratiques existantes. Cette recension vient compléter l'état des lieux sur les services en français dans le domaine de la DI en Ontario réalisé par Cardinal, Plourde

"...inclusion sociale des personnes présentant une DI dans le domaine des services et de l'emploi constituait un défi majeur en contexte francophone. " et Cobigo en 2017. Celui-ci a montré que l'inclusion sociale des personnes présentant une DI dans le domaine des services et de l'emploi constituait un défi majeur en contexte francophone. En Ontario, non seulement les services et les soutiens gouvernementaux pour les francophones présentant une DI manquent de visibilité, mais également nous avons constaté que le cadre législatif et règlementaire en matière d'intégration et des services en français, en raison de sa nature parcellaire, pouvait contribuer à créer des barrières systémiques à leur inclusion sociale. Le manque de considération pour cette population est également représenté dans l'absence de données à leur sujet au sein du gouvernement ontarien. Les francophones de l'Ontario présentant une DI font face à un cumul d'inégalités sociales, un enjeu qui se transpose aisément dans le domaine de l'intégration à l'emploi et des stratégies de soutien à concevoir pour cette population minoritaire. 
Le texte est divisé en trois sections. Dans un premier temps, nous proposons une définition sommaire de la DI et du domaine de la recherche portant sur le thème de l'inclusion sociale et de l'intégration à l'emploi des personnes présentant une DI. Dans un deuxième temps, nous précisons la méthode utilisée pour procéder à la recension des écrits. Dans un troisième temps, nous présentons nos résultats et esquissons des pistes d'analyse et des propositions d'élaboration de pratiques prometteuses dans le domaine de l'intégration à l'emploi.

\section{Qu'est-ce que la déficience intellectuelle?}

L'expression "déficience intellectuelle " (DI) sert à caractériser une personne qui présente un déficit des habiletés cognitives et adaptatives ayant apparu avant l'âge de 18 ans (American Association on Intellectual and Developmental Disabilities, 2013). Ce type de déficit peut limiter ces personnes sur les plans de la communication, des soins personnels, des compétences domestiques, des habiletés sociales et de l'autonomie. En Ontario, le Regroupement des partenaires francophones (RPF) explique que la DI «se traduit par une difficulté fondamentale d'apprentissage et de réalisation de certaines tâches quotidiennes. Elle peut avoir des retombées sur la capacité de réfléchir, de se concentrer,

"La DI n'est pas nécessairement synonyme de souffrance, mais les personnes qui ont ces limitations ont besoin d'appui...» d'exposer des idées, de raisonner ainsi que la mémoire" (RPF, 2017a). La DI n'est pas nécessairement synonyme de souffrance, mais les personnes qui ont ces limitations ont besoin d'appui, en particulier en ce qui a trait à leur inclusion sociale et communautaire. Pour sa part, le RPF est un carrefour de partenaires qui a pour mandat de promouvoir les intérêts et la qualité de vie des francophones présentant une DI ainsi que ceux de leurs familles. Parmi ses activités, le groupe sensibilise le public et les professionnels à la DI, appuie le développement de projets destinés à répondre aux besoins de cette population et fait de la consultation pour l'élaboration des services en français; ses activités ont pour objectif de contribuer à la surveillance de la qualité des services offerts aux francophones présentant une DI (RPF, 2017b).

Par " inclusion sociale des francophones présentant une DI ", nous entendons leur participation significative ou réelle à leur milieu de vie (Burchardt, Le Grand et Piachaud, 2002) et au travail (Llyod, Tse et Deane, 2006). L'inclusion sociale est associée au développement d'un sentiment d'appartenance à un ou plusieurs groupes et au fait de pouvoir participer à son épanouissement et vivre des relations sociales réciproques et 
valorisantes (Cobigo, et collab., 2016). C'est aussi de pouvoir accéder de façon équitable aux ressources et services offerts au sein d'une communauté (Cobigo, et collab., 2012).

"...l'étude de l'inclusion sociale sert à identifier les différents facteurs permettant à la personne présentant une DI d'accéder à des services..." Sur le plan analytique, l'étude de l'inclusion sociale sert à identifier les différents facteurs permettant à la personne présentant une DI d'accéder à des services, de pouvoir participer à son milieu ambiant et de développer un sentiment d'appartenance à sa communauté (Martin et Cobigo, 2011). Au Canada, Hall (2009) a mis en évidence l'importance de l'intégration à l'emploi comme étant un des six facteurs favorables à l'inclusion sociale des personnes handicapées (incluant les personnes présentant une DI). Les cinq autres facteurs sont : être accepté comme individu au-delà de son handicap; pouvoir vivre des relations personnelles; être engagé dans des activités sociales; avoir des conditions de vie adéquates; accéder à des formes de soutien formelles et informelles.

Toutefois, la recherche souligne que même les personnes les mieux intégrées à leur milieu peuvent aussi vivre de l'exclusion sociale (Hall, 2009; Abbott et McConkey, 2006; Lysaght, et collab., 2014; Lysaght, Cobigo et Hamilton, 2012; Randt, 2011). Entre autres, les personnes présentant une DI sont souvent moins susceptibles d'obtenir un emploi compétitif et rémunéré que les travailleurs au sein de la population générale (Hall et Kramer, 2009). Selon Statistique Canada, en 2006, seulement $25 \%$ des adultes présentant une DI avaient un emploi comparativement à $75 \%$ des adultes sans handicap (Statistique Canada, 2006). Il y avait aussi six fois plus d'adultes présentant une DI qui n'avaient jamais obtenu d'emploi comparativement aux adultes avec un autre handicap (p. ex. physique ou sensoriel) ou sans handicap (Statistique Canada, 2006; voir aussi Institute for Research and Development on Inclusion and Society, 2014).

Des statistiques plus récentes démontrent que les personnes présentant une DI en Ontario sont toujours moins susceptibles de participer au marché du travail (Lysaght, et collab., 2014) comparativement à la population générale canadienne (Turcotte, 2014). Selon un sondage réalisé par Lysaght, et collab. (2014), 38 \% des personnes présentant une DI ont déjà obtenu un emploi au salaire minimum, comparativement à $79 \%$ des Canadiens sans DI, selon Turcotte (2014). Cependant, ces emplois sont souvent de courte durée ou de nature sporadique - une observation qui correspond aux résultats plus récents de Lysaght, et collab. (2017) selon lesquels seulement $15 \%$ des personnes présentant une DI ont un emploi rémunéré au salaire minimum et plus. Lors de l'enquête, $24 \%$ de l'échantillon recevaient moins que le salaire minimum et $20 \%$ travaillaient sans 
rémunération. Les personnes présentant une DI qui sont âgées entre 25 et 34 ans, qui sont des hommes et qui habitent en milieu urbain sont plus susceptibles d'obtenir un emploi rémunéré au salaire minimum que les plus jeunes, les femmes et les personnes habitant en milieu rural (Lysaght, et collab., 2014). Enfin, la recherche montre que plus les

"...plus les personnes présentant une DI sont exclues du marché du travail, moins elles peuvent bénéficier des avantages reliés à l'emploi... » personnes présentant une DI sont exclues du marché du travail, moins elles peuvent bénéficier des avantages reliés à l'emploi, tels que l'autonomie financière et les sentiments d'estime de soi et d'appartenance à un milieu (Hall et Kramer, 2009; Ferrari, Nota et Soresi, 2008; Lysaght, Ouellette-Kuntz et Morrison, 2009).

Ces travaux constituent un point de départ incontournable pour l'étude des défis que vivent les personnes présentant une DI. Certains font preuve d'une sensibilité particulière aux enjeux de la différenciation sociale, tels que ceux de Lysaght, et collab. (2014) sur les distinctions selon le genre, l'âge ou le milieu de vie. Toutefois, dans la totalité des cas, les études sont uniquement en anglais. De plus, même s'ils prennent en compte les enjeux de la différenciation sociale, les travaux sur la DI n'opèrent aucune distinction entre les personnes présentant une DI qui appartiennent à un groupe minoritaire de celles qui font partie de la majorité. Il importe donc d'approfondir la situation des personnes présentant

"Le thème de l'intégration à l'emploi des francophones de l'Ontario présentant une DI constitue un point de départ valable et pertinent dans le contexte canadien." une DI qui appartiennent à ces groupes. Le thème de l'intégration à l'emploi des francophones de l'Ontario présentant une DI constitue un point de départ valable et pertinent dans le contexte canadien. Minorité de langue officielle reconnue par la Loi sur les services en français de l'Ontario, les francophones présentant une DI au sein de la province sont en droit de s'attendre à des services dans leur langue. Selon la définition inclusive utilisée par le gouvernement de l'Ontario, les francophones de la province représentent près de $5 \%$ de la population ou 611500 personnes sur une population de 13,5 millions d'habitants répartis dans cinq grandes régions géographiques : Est, Nord-Est, Nord-Ouest, Centre et Sud-Ouest. La plus forte proportion de francophones se trouve dans l'Est de la province, où ils constituent $42,2 \%$ de la population francophone de la province ou 257870 personnes, suivi du Centre, où ils représentent $30 \%$ de la population francophone ou 183605 personnes, et du Nord-Est, où ils représentent 20,8\% de la population francophone ou 127265 personnes. Dans le Sud-Ouest, ils composent 5,7 \% de la population francophone ou 35160 personnes et, dans le Nord-Ouest, ils forment 1,2\% de la population francophone ou 7610 personnes. Enfin, la majorité des francophones vit dans 26 régions désignées 
bilingues en vertu de la Loi sur les services en français de l'Ontario. À titre d'exemple, Ottawa, Toronto et Sudbury sont des régions désignées bilingues, ce qui signifie que le gouvernement de la province reconnaît qu'il a l'obligation d'offrir des services en français à sa population francophone vivant dans ces régions.

De plus, le contexte social et politique en Ontario s'avère propice à la prise en compte des préoccupations des personnes présentant une DI. À titre d'exemple, en 2014,

"...l'Assemblée législative de l'Ontario vote la création du Comité spécial des services aux personnes ayant une déficience intellectuelle. "
l'Assemblée législative de l'Ontario vote la création du Comité spécial des services aux personnes ayant une déficience intellectuelle. De surcroît, en 2016, l'Ombudsman de l'Ontario publie un rapport au titre évocateur, Dans l'impasse, dressant un état des lieux du système de soutien ontarien dans le domaine de la DI;

le rapport comprend 60 recommandations, dont celle de sensibiliser la population aux enjeux des personnes ayant une DI.

Rappelons que la Convention relative aux droits des personnes handicapées (2006) des Nations Unies énonce que les personnes handicapées ont le droit de vivre une pleine inclusion dans la communauté (article 19) et le droit de "gagner leur vie en accomplissant un travail librement choisi ou accepté sur un marché du travail et dans un milieu de travail ouverts, favorisant l'inclusion et accessibles aux personnes handicapées " (article 27). L'article 27 de la Convention énonce le droit «aux personnes handicapées d'avoir effectivement accès aux programmes d'orientation technique et professionnel, aux services de placement et aux services de formation professionnelle et continue offerts

"...la Charte canadienne des droits et libertés interdit toute forme de discrimination contre les personnes handicapées..." à la population en général ». Au Canada, la Charte canadienne des droits et libertés interdit toute forme de discrimination contre les personnes handicapées (article 15). En ce qui a trait à l'emploi, la Loi sur l'équité en matière d'emploi (1995) vise à réaliser l'égalité en milieu de travail et à réduire les désavantages subis par des minorités telles que les personnes présentant une DI (Gouvernement du Canada, 2017). Enfin, trois ministères fédéraux comprennent des objectifs dans le domaine de la déficience intellectuelle. Ce sont le ministère de l'Emploi et du Développement social, l'Agence du revenu du Canada et Santé Canada.

Bref, malgré les droits des personnes présentant une DI énoncés dans plusieurs documents, tels que la Convention relative aux droits des personnes handicapées (2006) et la Charte canadienne des droits et libertés, et la Loi sur l'équité en matière d'emploi (1995), 
la recherche courante démontre qu'il existe toujours des inégalités sociales importantes dans le domaine de l'intégration à l'emploi de ces personnes. Le présent article cherche à identifier les pratiques existantes et prometteuses en matière de formation professionnelle et d'intégration à l'emploi des personnes présentant une DI afin d'offrir des informations pratiques pour combattre ces inégalités.

\section{Méthode}

La recension des écrits a été effectuée afin d'identifier des pratiques prometteuses ou occasions de formation professionnelle et d'intégration à l'emploi pour des personnes présentant une DI. Deux questions ont guidé cette recherche : Quelles sont les pratiques prometteuses dans le domaine de la formation professionnelle des personnes présentant une déficience intellectuelle (DI)? Quelles sont les pratiques prometteuses pour faciliter le soutien et l'intégration à un emploi des personnes présentant une DI?

Les mots clés utilisés pour répondre à la première question sur les pratiques prometteuses dans le domaine de la formation professionnelle comprennent l'ensemble des termes pertinents reliés à la déficience intellectuelle (p. ex. déficience intellectuelle,

"...la formation professionnelle comprend l'ensemble des programmes, des services et des activités qui permettent aux personnes présentant une DI de faire l'apprentissage de capacités et d'aptitudes nécessaires..." déficience développementale, cognitive impairment) et tous ceux reliés à la formation professionnelle (p. ex. formation professionnelle, éducation, atelier, training, vocational education, vocational training, educational programs). En ce qui a trait à la deuxième question sur les pratiques prometteuses pour le soutien à l'emploi, nous avons utilisé les termes pertinents reliés à la DI et tous ceux reliés au soutien à l'emploi (p. ex. soutien à l'emploi, employabilité, supported employment, job coach). En simplifiant, nous pouvons dire que la formation professionnelle comprend l'ensemble des programmes, des services et des activités qui permettent aux personnes présentant une DI de faire l'apprentissage de capacités et d'aptitudes nécessaires à l'exercice d'un emploi (Potvin, Cardinal et Cobigo, 2017, p. 1). En ce qui a trait au soutien à l'intégration à un emploi, celui-ci est constitué de l'ensemble des programmes, des services et des activités en vue de faciliter l'employabilité des personnes présentant une DI (Potvin, Cardinal et Cobigo, 2017, p. 1). Les termes de recherche ont été identifiés par le thésaurus (une liste de sujets disponibles, dans une recherche avancée d'une base de données, qui correspondent aux mots clés). Nous avons aussi effectué la recherche au 
moyen de mots clés repérés dans les articles sur les mêmes sujets et en consultation avec un bibliothécaire de notre institution.

\section{Critères d'inclusion}

Afin d'être retenus, les articles devaient satisfaire aux six critères suivants :

1) porter sur une étude dont les participants présentent une DI;

2) concerner des participants qui sont d'âge adulte (18 ans et plus) ou qui ont le statut d'étudiant à l'école secondaire et sont en âge de transition vers des études postsecondaires ou vers le marché d'emploi;

3) être publiés en français ou en anglais;

4) provenir de revues scientifiques évaluées par des pairs;

5) être publiés depuis janvier 2000;

6) pouvoir être repérés au complet par Internet ou par la bibliothèque virtuelle de l'Université d'Ottawa.

\section{Sources des données}

Parmi les bases de données existantes en français et en anglais, nous avons consulté PsychINFO, ERIC, Business Source Complete et Érudit. Les bibliographies des articles pertinents identifiés grâce aux bases de données ont aussi été utilisées comme sources de données.

\section{Stratégie de sélection d'articles}

La sélection des articles a été effectuée en sept étapes :

"Nous avons procédé à la révision des titres d'articles à l'intérieur des bases de données existantes..."
1) Nous avons procédé à la révision des titres d'articles à l'intérieur des bases de données existantes en excluant les articles non pertinents, selon les critères d'inclusion énumérés ci-dessus;

2) Nous avons révisé les résumés des articles à l'intérieur des bases de données en excluant ceux qui nous sont apparus non pertinents;

3) Nous avons exporté l'ensemble des articles retenus dans le programme Zotéro;

4) Nous avons fait la révision préliminaire des articles et exclu les articles non pertinents;

5) Nous avons repris les articles sélectionnés pour en faire une analyse plus détaillée; 
6) Nous avons fait l'analyse des bibliographies des articles sélectionnés afin de repérer tout autre article approprié;

7) Nous avons produit des tableaux qui résument le contenu de chacun des articles sélectionnés.

Ces tableaux ont servi à organiser les informations reliées aux questions de recherche, à élaborer des propositions de pratiques prometteuses et à déterminer les niveaux de preuve scientifique des données retenues. Nous présentons ici des données qui reposent sur les meilleures preuves scientifiques que nous avons identifiées selon la gradation des recommandations proposée par la Haute autorité de santé (2016) et selon les consignes de Melnyk et Fineout-Overholt (2011).

Le niveau de preuve d'une étude caractérise la capacité de l'étude à répondre à la question posée. La capacité d'une étude à répondre à la question posée est jugée sur la correspondance de l'étude au cadre du travail (question, population, critères de jugement) et sur les caractéristiques suivantes : l'adéquation du protocole d'étude à la question posée; l'existence ou non de biais importants dans la réalisation; l'adaptation de l'analyse statistique aux objectifs de l'étude; la puissance de l'étude et en particulier la taille de l'échantillon (Haute autorité de santé, 2013).

"...la majorité des informations incluses provient d'études qualitatives et descriptives..."

Notons toutefois que la majorité des informations incluses provient d'études qualitatives et descriptives et donc offre un faible niveau de preuve scientifique (niveau 6-C). D'ailleurs, l'étude de Grigal, Hart et Migliore (2011) démontre une présomption scientifique (niveau 4-B) provenant d'une méthode d'analyse statistique avec groupe de comparaison. Il faut donc interpréter avec prudence la force des recommandations basée sur les niveaux de preuve scientifique au sein de la littérature. Comme le souligne la Haute autorité de santé :

Cette gradation des recommandations fondée sur le niveau de preuve scientifique de la littérature venant à l'appui de ces recommandations ne présume pas obligatoirement du degré de force de ces recommandations. En effet, il peut exister des recommandations de grade $\mathrm{C}$ ou fondées sur un accord d'experts néanmoins fortes malgré l'absence d'un appui scientifique (2013, p. 8). 

formation professionnelle et l'intégration à un emploi des personnes présentant une DI

\begin{tabular}{|c|c|c|c|c|c|}
\hline $\begin{array}{l}\text { Auteurs et années } \\
\text { (voir la bibliographie } \\
\text { pour les références } \\
\text { complètes des } \\
\text { textes) }\end{array}$ & $\begin{array}{l}\text { Lieux de } \\
\text { l'étude }\end{array}$ & Participants & Méthodes utilisées & Conclusions principales & $\begin{array}{l}\text { Niveau de } \\
\text { preuve } \\
\text { scientifique }\end{array}$ \\
\hline $\begin{array}{l}\text { JUDGE, Sharon, et } \\
\text { Dolores Izuzquiza } \\
\text { GASSET (2015) }\end{array}$ & $\begin{array}{c}\text { Madrid } \\
\text { (Espagne) }\end{array}$ & $\begin{array}{l}\text { Étudiants présentant une } \\
\text { DI âgés de } 18 \text { à } 30 \text { ans; } \\
n=60\end{array}$ & Description d'un programme & $\begin{array}{l}\text { Parmi les composantes indispensables } \\
\text { pour l'inclusion à l'emploi des étudiants } \\
\text { présentant une DI, les auteures } \\
\text { suggèrent le besoin d'une approche } \\
\text { individualisée et centrée sur la personne, } \\
\text { le stage et le mentorat par les pairs. }\end{array}$ & $6-\mathrm{C}$ \\
\hline $\begin{array}{l}\text { HART BARNETT, } \\
\text { Juliet E., et Robert } \\
\text { CRIPPEN (2014) }\end{array}$ & États-Unis & $\begin{array}{l}\text { Étudiants d'école } \\
\text { secondaire présentant une } \\
\text { DI ou/et un TSA; } n=\text { pas } \\
\text { de données disponibles }\end{array}$ & Description d'un programme & $\begin{array}{l}\text { Les auteurs proposent une approche } \\
\text { comprenant huit étapes pour } \\
\text { l'enseignement et la formation } \\
\text { professionnelle d'étudiants présentant } \\
\text { une DI en milieu scolaire. }\end{array}$ & $6-C$ \\
\hline $\begin{array}{l}\text { DUMAIS, Lucie, } \\
\text { Alexandra PROHET et } \\
\text { Léonie ARCHAMBAULT } \\
\text { (2012) }\end{array}$ & $\begin{array}{l}\text { Québec } \\
\text { (Canada) }\end{array}$ & $\begin{array}{l}\text { Personnes présentant } \\
\text { des troubles du spectre } \\
\text { autistique (TSA), réparties } \\
\text { en neuf cohortes; } n=45\end{array}$ & $\begin{array}{l}\text { Évaluation de programme; } \\
\text { étude longitudinale avec des } \\
\text { mesures de type avant- } \\
\text { après (observations in situ } \\
\text { et entrevues semi-dirigées); } \\
\text { triangulation des données } \\
\text { des participants, de leurs } \\
\text { familles, d'intervenants et } \\
\text { d'employeurs }\end{array}$ & $\begin{array}{l}\text { Les auteures proposent un modèle } \\
\text { d'intervention comprenant quatre étapes } \\
\text { pour l'intégration à l'emploi. Les quatre } \\
\text { étapes sont : l'évaluation du participant; } \\
\text { la formation; la recherche d'emploi; } \\
\text { l'intégration en emploi et le suivi à court } \\
\text { ou à moyen terme. Le modèle est testé } \\
\text { dans le cadre d'un projet pilote qui a } \\
\text { vu } 50 \% \text { de ses participants obtenir un } \\
\text { emploi, ce qui démontre que le modèle } \\
\text { d'intervention proposé s'avère pertinent. }\end{array}$ & $6-C$ \\
\hline
\end{tabular}




\begin{tabular}{|c|c|c|c|c|c|}
\hline $\begin{array}{l}\text { KLEINERT, Harold L., } \\
\text { et collab. (2012) }\end{array}$ & $\begin{array}{c}\text { Kentucky } \\
\text { (États-Unis) }\end{array}$ & $\begin{array}{l}\text { Étudiants présentant une } \\
\text { DI; } n=7\end{array}$ & Description d'un projet pilote & $\begin{array}{l}\text { Les auteurs étudient un modèle } \\
\text { d'éducation postsecondaire inclusif dans } \\
\text { le cadre d'un projet pilote et suggèrent } \\
\text { d'adopter une approche individualisée et } \\
\text { centrée sur la personne, que le mentorat } \\
\text { est indispensable, et que l'aspect } \\
\text { social de l'institution d'enseignement } \\
\text { est tout aussi important que son volet } \\
\text { académique. }\end{array}$ & $6-\mathrm{C}$ \\
\hline $\begin{array}{l}\text { GRIGAL, Meg, } \\
\text { Debra HART et Alberto } \\
\text { MIGLIORE (2011) }\end{array}$ & États-Unis & $\begin{array}{l}\text { Étudiants présentant } \\
\text { une DI qui ont reçu des } \\
\text { services d'éducation } \\
\text { spécialisés, âgés de } 13 \\
\text { à } 16 \text { ans en décembre } \\
2000 ; \text { au moment des } \\
\text { analyses : } \\
19 \text { à } 23 \text { ans; } n=520 \\
\text { Groupe de comparaison : } \\
\text { étudiants présentant } \\
\text { d'autres handicaps }\end{array}$ & $\begin{array}{l}\text { Analyses secondaires } \\
\text { du National Longitudinal } \\
\text { Transition Survey } 2 \text { (NLTS- } \\
\text { 2); statistiques descriptives }\end{array}$ & $\begin{array}{l}\text { Selon les auteurs, les étudiants } \\
\text { présentant une DI qui ont effectué des } \\
\text { études postsecondaires ont plus de } \\
\text { probabilités d'obtenir un emploi que ceux } \\
\text { qui n'ont pas eu accès à de telles études. } \\
\text { Les auteurs soulignent l'importance } \\
\text { des études postsecondaires dans le } \\
\text { parcours des étudiants présentant une } \\
\text { Dl au lieu de les diriger directement vers } \\
\text { des programmes de soutien à l'emploi, } \\
\text { des entreprises de travail adapté et des } \\
\text { programmes de jour pour adultes. }\end{array}$ & $4-B$ \\
\hline $\begin{array}{l}\text { CARROLL, Stuart Z., } \\
\text { E. Richard BLUMBERG } \\
\text { et Jerry G. PETROFF } \\
\text { (2008) }\end{array}$ & $\begin{array}{l}\text { New Jersey } \\
\text { (États-Unis) }\end{array}$ & $\begin{array}{l}\text { Étudiants présentant une } \\
\text { DI âgés de } 18 \text { à } 25 \text { ans } \\
\text { au début du programme; } \\
n=\text { pas de données } \\
\text { disponibles }\end{array}$ & \begin{tabular}{|l|} 
Évaluation de programme \\
(taux d'emploi des \\
participants, leur autonomie, \\
la participation en vie \\
communautaire, les \\
relations sociales, etc.); \\
aucune statistique proposée
\end{tabular} & $\begin{array}{l}\text { Les auteurs mettent l'accent sur } \\
\text { l'importance du mentorat des étudiants } \\
\text { présentant une DI par des pairs sans } \\
\text { DI, l'importance aussi des rapports de } \\
\text { collaboration entre les enseignants, les } \\
\text { étudiants, le personnel administratif, } \\
\text { les familles et les ressources } \\
\text { communautaires; accent aussi sur le } \\
\text { rôle des institutions postsecondaires. Les } \\
\text { auteurs proposent des lignes directrices } \\
\text { pour les éducateurs afin d'améliorer } \\
\text { l'enseignement en classe. }\end{array}$ & $6-C$ \\
\hline
\end{tabular}




\begin{tabular}{|c|c|c|c|c|c|}
\hline $\begin{array}{l}\text { Auteurs et années } \\
\text { (voir la bibliographie } \\
\text { pour les références } \\
\text { complètes des } \\
\text { textes) }\end{array}$ & $\begin{array}{l}\text { Lieux de } \\
\text { l'étude }\end{array}$ & Participants & Méthodes utilisées & Conclusions principales & $\begin{array}{l}\text { Niveau de } \\
\text { preuve } \\
\text { scientifique }\end{array}$ \\
\hline WEIR, Cate (2004) & $\begin{array}{l}\text { États-Unis } \\
\text { (État dans } \\
\text { le nord-est) }\end{array}$ & \begin{tabular}{|l|} 
Étudiants d'institution \\
postsecondaire présentant \\
une $\mathrm{DI} ; \mathrm{n}=8$
\end{tabular} & $\begin{array}{l}\text { Observation des } \\
\text { participants; notes prises } \\
\text { sur le terrain }\end{array}$ & $\begin{array}{l}\text { L'auteure montre que le soutien } \\
\text { individualisé et flexible de chaque } \\
\text { étudiant qui est dirigé par ses buts } \\
\text { et vœux, est un ingrédient essentiel } \\
\text { pour la réussite en milieu collégial. La } \\
\text { collaboration des partenaires (collège, } \\
\text { agences, famille, amis) est aussi } \\
\text { essentielle. }\end{array}$ & $6-C$ \\
\hline $\begin{array}{l}\text { DOLYNIUK, Chrystina } \\
\text { A., et collab. (2002) }\end{array}$ & $\begin{array}{l}\text { New Jersey } \\
\text { (États-Unis) }\end{array}$ & $\begin{array}{l}\text { Étudiants présentant une } \\
\text { DI, âgés de } 16 \text { à } 20 \text { ans; } \\
n=17 \\
n=23 \text { pairs sans DI } \\
\text { (mentors) }\end{array}$ & $\begin{array}{l}\text { Description d'un projet } \\
\text { pilote; journaux de bord } \\
\text { d'étudiants et de leur } \\
\text { mentor; notes prises sur } \\
\text { le terrain; questionnaires; } \\
\text { anecdotes }\end{array}$ & $\begin{array}{l}\text { Les auteures montrent l'avantage du } \\
\text { mentorat pour l'apprentissage et le } \\
\text { développement d'habiletés sociales. }\end{array}$ & $6-C$ \\
\hline $\begin{array}{l}\text { MAUTZ, Denise, } \\
\text { Keith STOREY et Nick } \\
\text { CERTO (2001) }\end{array}$ & $\begin{array}{c}\text { Californie } \\
\text { (États-Unis) }\end{array}$ & $\begin{array}{l}\text { Personne de } 40 \text { ans } \\
\text { présentant une DI sévère; } \\
n=1\end{array}$ & Observations directes & $\begin{array}{l}\text { Les auteurs confirment que l'utilisation } \\
\text { d'un appareil de communication, le } \\
\text { soutien d'un mentor qui a reçu une } \\
\text { formation spécialisée et le soutien des } \\
\text { collègues sont nécessaires pour accroitre } \\
\text { le niveau d'interactions sociales au } \\
\text { travail. }\end{array}$ & $6-C$ \\
\hline $\begin{array}{l}\text { HALL, Maeda, Harold } \\
\text { L. KLEINERT et } \\
\text { Jacqueline Farmer } \\
\text { KEARNS (2000) }\end{array}$ & $\begin{array}{c}\text { Kentucky } \\
\text { (États-Unis) }\end{array}$ & $\begin{array}{l}\text { Étudiants présentant une } \\
\text { Dl âgés de } 18 \text { à } 21 \text { ans; } \\
n=14\end{array}$ & $\begin{array}{l}\text { Évaluation de programme; } \\
\text { observations }\end{array}$ & $\begin{array}{l}\text { Les auteurs montrent que le programme } \\
\text { de transition vers le collège étudié a } \\
\text { contribué à l'élargissement du réseau } \\
\text { social des étudiants et à une meilleure } \\
\text { insertion communautaire par l'entremise } \\
\text { d'apprentissages par les pairs (sans DI). }\end{array}$ & $6-\mathrm{C}$ \\
\hline
\end{tabular}




\section{Résultats}

Nous avons retenu dix articles empiriques sur le sujet de la formation professionnelle et de l'intégration à l'emploi des personnes présentant une DI, dont un seulement en français (Hart Barnett et Crippen, 2014; Carroll, Blumberg et Petroff, 2008; Dolyniuk, et collab., 2002; Dumais, Prohet et Archambault, 2012; Grigal, Hart et Migliore, 2011; Hall, Kleinert et Kearns, 2000; Judge et Gasset, 2015; Kleinert, et collab., 2012; Mautz, Storey et Certo, 2001; Weir, 2004).

De façon générale, les écrits servent à souligner le rôle clé des institutions postsecondaires, telles que les collèges et les universités, dans l'acquisition des habiletés interpersonnelles et professionnelles nécessaires à l'intégration à un emploi des personnes présentant une DI (Grigal, Hart et Migliore, 2011; Hart Barnett et Crippen, 2014; Kleinert, et collab., 2012; Carroll, Blumberg et Petroff, 2008; Dolyniuk, et collab.,

"La recherche dans le domaine de la formation professionnelle invite aussi les institutions à privilégier une approche individualisée..." 2002; Hall, Kleinert et Kearns, 2000). La recherche dans le domaine de la formation professionnelle invite aussi les institutions à privilégier une approche individualisée et centrée sur la personne (Judge et Gasset, 2015; Dumais, Prohet et Archambault, 2012; Kleinert, et collab., 2012; Carroll, Blumberg et Petroff, 2008; Weir, 2004; Dolyniuk, et collab., 2002). De façon plus spécifique, une telle approche devrait donner aux étudiants l'occasion d'apprendre un contenu académique approprié à leur âge (Grigal, Hart et Migliore, 2011; Kleinert, et collab., 2012; Carroll, Blumberg et Petroff, 2008; Weir, 2004; Dolyniuk, et collab., 2002; Hall, Kleinert et Kearns, 2000) dans un contexte favorisant les échanges avec leurs pairs sans DI.

Pour leur part, Judge et Gasset (2015) soulignent la pertinence d'offrir des stages comme stratégie pour l'intégration à un emploi régulier. Plusieurs confirment aussi l'intérêt de jumeler des étudiants présentant une DI avec leurs pairs sans DI (Judge et Gasset, 2015; Kleinert, et collab., 2012; Carroll, Blumberg et Petroff, 2008; Dolyniuk, et collab., 2002).

"Les écrits mettent également l'accent sur les rapports de collaboration entre les acteurs clés $d u$ milieu...»
Les écrits mettent également l'accent sur les rapports de collaboration entre les acteurs clés du milieu, soit la famille, les amis, les pairs dans le milieu scolaire, les spécialistes du soutien à l'intégration professionnelle, les accompagnateurs, les conseils scolaires locaux, le personnel administratif scolaire, les employeurs, les ressources communautaires et les 
institutions postsecondaires. Ces différents acteurs favorisent l'inclusion des personnes présentant une DI dans la communauté (p. ex. les milieux scolaires et professionnels) (Hart Barnett et Crippen, 2014; Dumais, Prohet et Archambault, 2012; Kleinert, et collab., 2012; Carroll, Blumberg et Petroff, 2008; Weir, 2004; Dolyniuk, et collab., 2002; Hall, Kleinert et Kearns, 2000).

La recherche suggère que les étudiants qui participent à un programme d'éducation inclusif et individualisé offert par une institution postsecondaire (p. ex. un collège) ainsi qu'à des ateliers de formation professionnelle dans la communauté obtiennent de meilleurs taux d'employabilité ( $26 \%$ plus probable) comparativement à ceux qui ont uniquement participé à des ateliers de formation professionnelle (sans une formation

"En moyenne, les personnes ayant reçu une formation auraient aussi un revenu supérieur..." postsecondaire) (Kleinert, et collab., 2012). En moyenne, les personnes ayant reçu une formation auraient aussi un revenu supérieur de $73 \%$ à celui des personnes qui n'ont pas accédé à un programme d'éducation postsecondaire (Kleinert, et collab., 2012).

Enfin, de tous les articles repérés, nous avons retenu celui de Dumais, Prohet et Archambault (2012), car, au lieu de s'en tenir à une variable en particulier, les auteures systématisent les différentes étapes à suivre en vue de l'intégration à l'emploi incluant la formation professionnelle. Leur recherche, qui portait sur des étudiants présentant des troubles du spectre de l'autisme (TSA), invite à procéder en quatre étapes, chacune comprenant des activités précises. Il est important de noter, cependant, que les personnes ayant un TSA ne présentent pas toutes également une DI. Ces étapes comprennent :

1) l'évaluation;

2) la formation;

3) la recherche d'emploi;

4) l'intégration à un emploi.

Selon Dumais, Prohet et Archambault (2012), dans un premier temps, il faut effectuer l'évaluation individuelle de la personne afin de bien identifier ses intérêts, désirs, rêves, préférences, besoins et objectifs afin de maximiser son encadrement (voir aussi Judge et Grasset, 2015; Carroll, Blumberg et Petroff, 2008; Weir, 2004; Dolyniuk, et collab., 2002; Hall, Kleinert et Kearns, 2000; Kleinert, et collab., 2012). Les auteures suggèrent aussi de créer un plan d'action personnalisé, ou régime d'enseignement individualisé et d'emploi, pour chaque personne. Ce plan devrait énoncer clairement les objectifs sociaux, académiques et professionnels de l'étudiant ainsi que les étapes à suivre pour les atteindre. 
Afin de favoriser l'autonomie et l'autodétermination des étudiants présentant une DI, Dumais, Prohet et Archambault (2012) recommandent aussi que ces derniers soient invités à collaborer au processus de planification de leur formation et de leur évaluation (voir aussi Hart Barnett et Crippen, 2014; Carroll, Blumberg et Petroff, 2008; Weir, 2004; Dolyniuk, et collab., 2002; Kleinert, et collab., 2012).

Dans un deuxième temps, à l'étape plus spécifique de la formation, Dumais, "...Dumais, Prohet et Archambault (2012) proposent des stratégies d'enseignement interactif dans un environnement de travail typique..." Prohet et Archambault (2012) proposent des stratégies d'enseignement interactif dans un environnement de travail typique, au lieu d'un enseignement magistral, afin de favoriser l'acquisition et le transfert des connaissances (voir aussi Hart Barnett et Crippen, 2014; Dolyniuk, et collab., 2002; Hall, Kleinert et Kearns, 2000). Hart Barnett et Crippen (2014) ont aussi trouvé que la création de manuels d'enseignement et de formation comprenant des supports visuels est très utile, car les étudiants présentant une DI peuvent ensuite y faire référence lorsqu'ils ont un emploi.

Parmi les activités de soutien au développement des habiletés pratiques des étudiants dans le cadre de leur formation, la recherche invite à enseigner la création du curriculum vitae, la préparation à une entrevue de travail et la gestion d'un budget (Hart Barnett et Crippen, 2014; Hall, Kleinert et Kearns, 2000). En ce qui a trait aux habiletés sociales, les travaux mettent l'accent sur les habiletés interpersonnelles telles que la communication professionnelle (Dumais, Prohet et Archambault, 2012; Hart Barnett et Crippen, 2014;

"Parmi les activités plus académiques, il faut aussi compter sur le développement de compétences d'écriture et de lecture..." et Petroff, 2008). Judge et Gasset, 2015; Carroll, Blumberg et Petroff, 2008; Dolyniuk, et collab., 2002; Hall, Kleinert et Kearns, 2000). Parmi les activités plus académiques, il faut aussi compter sur le développement de compétences d'écriture et de lecture, mais aussi les mathématiques (Hart Barnett et Crippen, 2014; Judge et Gasset, 2015; Carroll, Blumberg

Dans un troisième temps, vient l'étape de la recherche d'emploi pour les personnes présentant une DI. Dumais, Prohet et Archambault (2012) considèrent important de sensibiliser les employeurs et les employés au sein de la communauté à l'employabilité des personnes ayant une DI. Cette invitation vise à élargir le bassin d'employeurs potentiels et à encourager le placement des personnes présentant une DI en stage au sein d'entreprises situées dans le milieu. Dumais, Prohet et Archambault (2012) mettent l'accent sur le 
développement de l'autonomie de la personne lors de cette étape, c'est-à-dire que tout en étant soutenue, la personne présentant une DI est encouragée à procéder à une démarche autonome de recherche d'emploi.

Dans un quatrième temps, à l'étape de l'intégration à l'emploi, Dumais, Prohet et Archambault (2012) soulignent que le milieu d'emploi lui-même doit être ouvert et

"...le milieu d'emploi luimême doit être ouvert et conscient des forces et des besoins d'un nouveau collègue présentant une DI, ainsi que des enjeux reliés à la DI..." conscient des forces et des besoins d'un nouveau collègue présentant une DI, ainsi que des enjeux reliés à la DI (p. ex. la période d'insertion en emploi peut être plus longue pour une personne présentant une DI). Il devient important, pour favoriser l'intégration à l'emploi, que des agents d'intégration ou accompagnateurs en milieu de travail soient embauchés ou identifiés sur place afin de soutenir les personnes présentant une DI. Pour Dumais, Prohet et Archambault (2012), ces personnes peuvent servir de points de repères immédiats en cas de problème ou de défi après l'intégration en emploi (voir aussi Dolyniuk, et collab., 2002). Elles ont aussi la possibilité de favoriser les interactions sociales au travail (Mautz, Storey et Certo, 2001).

\section{Discussion}

Il existe très peu de données probantes sur les pratiques prometteuses ainsi que les avantages et les défis de l'intégration à un emploi des personnes présentant une DI et, surtout, en contexte francophone. Cependant, lorsque nous combinons les travaux

"...nous pouvons identifi-

er trois étapes à prendre en compte en vue de favoriser l'intégration à un emploi de cette population..." présentés ci-dessus, nous pouvons identifier trois étapes à prendre en compte en vue de favoriser l'intégration à un emploi de cette population, où chaque étape comporte des activités recommandées dans les travaux scientifiques. Ainsi, l'inclusion en milieux scolaires postsecondaires, le mentorat et l'insertion en stage de travail en milieux communautaires ressortent comme des stratégies prometteuses dans le domaine de l'inclusion sociale.

\section{Le stage et le mentorat : des pratiques prometteuses}

Une conclusion inspirante qui ressort des écrits a trait à la mise en place de stages et d'activités de mentorat comme condition favorable à l'intégration à l'emploi et comme levier d'inclusion sociale. Soulignons que de telles pratiques caractérisent aussi le 
travail de certains organismes francophones en Ontario, en particulier l'Association pour l'intégration sociale d'Ottawa (AISO), qui joue un rôle important dans le milieu francophone d'Ottawa auprès des jeunes francophones présentant une DI. L'organisme collabore de façon étroite avec les institutions de formation comme la Cité, sise également à Ottawa, pour favoriser l'intégration à l'emploi des francophones présentant une DI.

"Lorsque l'on systématise les différentes étapes en vue de l'intégration à l'emploi des personnes présentant une $D I$, la recherche montre que celles-ci sont en contact avec des animateurs..."

Lorsque l'on systématise les différentes étapes en vue de l'intégration à l'emploi des personnes présentant une DI, la recherche montre que celles-ci sont en contact avec des animateurs, d'autres étudiants, des formateurs, des employeurs et des intervenants. Elles doivent développer des rapports de collaboration étroits avec ces personnes à chaque étape de leur parcours. Chaque étape permet ainsi de constater que le système de soutien est complexe. Il ressort des écrits que le stage en milieu de travail est une pratique particulièrement prometteuse pour permettre aux personnes présentant une DI d'intégrer les différentes activités dont elles doivent faire l'apprentissage au moment de leur formation. Grâce au stage, l'étudiant est intégré au sein d'une entreprise dans le cadre de sa formation professionnelle afin d'acquérir des expériences de travail.

De façon plus précise, les écrits sur l'employabilité des personnes présentant une DI soulignent que le système scolaire actuel ne suffit pas à préparer cette population pour le marché du travail (Lysaght, et collab., 2012; Bouck, 2004; Patton, et collab., 1996). Selon Lysaght, et collab. (2012), des programmes études-emploi, soit des programmes qui offrent des occasions de stages, peuvent faire vivre des expériences de travail inestimables aux personnes présentant une DI (voir aussi Beyer et Kaehne, 2008; Kaehne et Beyer, 2013; Vilà, Pallisera et Fullana, 2007). En effet, Judge et Gasset (2015) ont constaté que

"...le stage constitue une activité de formation professionnelle fondamentale pour les personnes présentant une DI. » le stage constitue une activité de formation professionnelle fondamentale pour les personnes présentant une DI. Le modèle du stage offre à ces personnes une occasion de soutien adapté à leurs besoins et à leurs intérêts afin de leur permettre de développer leur autonomie et leurs habiletés liées à l'emploi (Judge et Gasset, 2015; Kaehne et Beyer, 2013). Cependant, le soutien offert pendant la période de stage n'est pas suffisant en soi; afin d'être efficace à long terme, ce soutien doit être continu et provenir de divers milieux, scolaire ou de travail (Beyer et Kaehne, 2008; Judge et Gasset, 2015). 
Les chercheurs suggèrent aussi de maximiser le stage par le moyen du mentorat (Judge et Gasset, 2015; Kaehne et Beyer, 2013). Les études dans le domaine de la DI conceptualisent le mentorat comme un partenariat, c'est-à-dire comme une relation dyadique où deux personnes (un mentor et un mentoré) s'engagent dans une relation de soutien (Judge et Gasset, 2015; Kleinert, et collab., 2012; Carroll, Blumberg et Petroff,

"...la recherche révèle aussi quil existe une multiplicité de relations de soutien qui caractérisent l'encadrement d'une personne présentant une DI au moment de sa formation professionnelle et de son intégration à un emploi. » 2008; Dolyniuk, et collab., 2002). Cependant, la recherche révèle aussi qu'il existe une multiplicité de relations de soutien qui caractérisent l'encadrement d'une personne présentant une DI au moment de sa formation professionnelle et de son intégration à un emploi (p. ex. le corps professoral, les pairs, les accompagnateurs en milieu de travail, la personne de référence au travail et l'employeur) (Hart Barnett et Crippen, 2014; Dumais, Prohet et Archambault, 2012; Kleinert, et collab., 2012; Carroll, Blumberg et Petroff, 2008; Weir, 2004; Dolyniuk, et collab., 2002; Hall, Kleinert et Kearns, 2000). Le mentorat constitue une composante essentielle du stage en emploi. Il peut prendre plusieurs formes, selon le milieu. De plus, le mentor peut être une personne différente, selon que l'on est en milieu scolaire ou au travail.

La recherche révèle que le mentorat est à la base de plusieurs programmes d'éducation postsecondaire et de formation professionnelle pour les personnes présentant une DI (Culnane, Eisenman et Murphy, 2016; Giust et Valle-Riestra, 2017; Jones et Goble, 2012; Kaehne et Beyer, 2013; Lindsay, Hartman et Fellin, 2016). De plus, une recherche systématique dans les écrits montre que l'on accorde beaucoup d'importance à la valeur du mentorat en contexte de transition des personnes présentant un handicap vers les études postsecondaires et vers l'emploi (Lindsay, Hartman et Fellin, 2016).

Sans parler directement du mentorat, plusieurs études soulignent aussi les avantages reliés au jumelage d'étudiants présentant une DI avec d'autres étudiants sans DI (Carroll, Blumberg et Petroff, 2008; Dolyniuk, et collab., 2002; Dumais, Prohet et Archambault,

"Les travaux sur le mentorat contiennent peu d'informations spécifiques à la DI et ne contiennent aucune information sur les populations francophones... "
2012; Hall, Kleinert et Kearns, 2000; Judge et Gasset, 2015; Kleinert, et collab., 2012; Weir, 2004). Cependant, Kaehne et Beyer (2013) constatent le besoin d'approfondir la recherche sur cette question. De plus, il est important de noter que la majorité des études à partir desquelles nous balisons nos recherches sur les francophones présentant une DI démontre un faible 
niveau de preuve scientifique. Les travaux sur le mentorat contiennent peu d'informations spécifiques à la DI et ne contiennent aucune information sur les populations francophones (Lindsay, Hartman et Fellin, 2016). Ceux de Dumais, Prohet et Archambault (2012) sur les TSA sont inspirants et sont en français, mais ils portent sur des personnes présentant un TSA. Ces personnes n'ont pas forcément une DI; ces individus (TSA sans DI) n'auront pas les mêmes forces et défis que les personnes présentant une DI en absence d'un TSA, ou celles présentant une DI et un TSA. D'ailleurs, nous constatons que l'hétérogénéité de la population étudiante présentant une DI représente en soi un défi pour la formation professionnelle et l'intégration à emploi. Pour cette raison, nous convenons que les stratégies proposées doivent être flexibles afin qu'elles puissent être adaptées aux besoins et aux forces uniques de chaque personne.

Les résultats de cette recension invitent à poursuivre la recherche sur la question des stratégies prometteuses dans le domaine de la formation professionnelle et de l'intégration à l'emploi. La plupart des travaux portant sur des populations vivant aux États-Unis, sauf exception, il existe un besoin criant de données sur la situation au Canada. De plus,

les travaux présentés ne portent pas sur des populations vivant en situation minoritaire. " les travaux présentés ne portent pas sur des populations vivant en situation minoritaire. Or, cette réalité, même si elle n'est pas unique au Canada, confere à la recherche une particularité qui invite à une sensibilité accrue aux effets de la langue sur le développement de pratiques prometteuses.

\section{Conclusion}

Cette présentation des travaux sur l'inclusion sociale des personnes présentant une DI, en particulier sur l'intégration à l'emploi et la formation professionnelle, a permis de mettre en lumière les principales conclusions et recommandations des auteures et auteurs en vue de favoriser le développement de pratiques prometteuses. Malgré leurs limites, nous tirons d'importantes leçons de ces travaux en vue de poursuivre la recherche sur la question en milieu francophone en Ontario. Nous pouvons conclure sans ambages que la recherche sur les personnes présentant une DI est nettement insuffisante en ce qui concerne l'un des leviers principaux de l'inclusion sociale : l'intégration à l'emploi. Ces travaux nous paraissent également nécessaires pour élaborer des stratégies de soutien à l'emploi pour cette population minoritaire. Il importe aussi de mieux comprendre les possibilités de stages et de mentorat en français dans les milieux de travail pour les francophones de l'Ontario présentant une DI. 
Par contre, nous convenons que les défis sont de taille lorsque vient le temps d'étudier des populations comme celles présentant une DI dans un contexte minoritaire, si ce n'est sur le plan démographique. En effet, selon un courriel qui nous a été transmis par le ministère des Services sociaux et communautaires (MSSC) le 27 juin 2017, en 2015 et en 2016, environ 440 personnes présentant une DI ont reçu des services de soutien en établissement en français. En 2017, environ $4 \%$ des personnes qui ont complété une évaluation pour recevoir des services pour personnes présentant une DI, dans l'un des Services de l'Ontario pour les personnes ayant une déficience intellectuelle (SOPDI) en Ontario, ont déclaré le français comme langue parlée (MSSC, 2017). Cependant, ces données ne portent pas directement sur le nombre de personnes francophones présentant une DI en Ontario, puisqu'elles proviennent d'évaluations complétées pour de nouvelles demandes de services. Enfin, parmi les 63743 usagers des SOPDI, 807 personnes ont indiqué le français comme langue préférée de correspondance (environ

"...certaines personnes présentant une DI en Ontario ne reçoivent pas de services des SOPDI ou autres agences de services spécialisés... "

$1 \%)$. Il est important de souligner que certaines personnes présentant une DI en Ontario ne reçoivent pas de services des SOPDI ou autres agences de services spécialisés, et ne sont pas connues par le MSSC comme ayant une DI (Lin, et collab., 2013). Non seulement ces données sont très parcellaires, mais également elles témoignent de l'invisibilité des francophones présentant une DI dans le système de soins et de soutien. Au terme de cette étude, nous pouvons réaffirmer sans hésiter que le besoin de plus de travaux dans ce domaine particulier s'avère criant.

\section{Bibliographie}

ABBOTT, Suzanne, et Roy MCCONKEY (2006). «The barriers to social inclusion as perceived by people with intellectual disabilities ", Journal of Intellectual Disabilities, Vol. 10, № 3, p. 275-287.

AMERICAN ASSOCIATION ON INTELLECTUAL AND DEVELOPMENTAL DISABILITIES (2013). " Definition of intellectual disability ", réf. du 15 septembre 2017, http://aaidd.org/intellectual-disability/definition\#.VH3S9THF86w

BÉLANGER, Nathalie, Mona PARÉ et Marie-Eve GAGNÉ (2017). «École, handicap et francophonie : comment pallier les inégalités au sein d'un groupe minoritaire? ", Canadian Journal of Disability Studies, Vol. 6, № 2, p. 45-77, DOI :10.15353/cjds.v6i2.350. 
BÉLANGER, Nathalie, et collab. (2015). « Les enfants en situation de handicap sévère dans la région d'Ottawa ", Revue de la littérature et état des lieux des services et des ressources accessibles à la minorité francophone, Ottawa, Université d'Ottawa, Chaire de recherche francophonie et éducation, 56 p.

BÉLANGER, Nathalie, et Hermann DUCHESNE (2010). Des écoles en mouvement. Inclusion d'élèves en situation de handicap ou éprouvant des difficultés à l'école, Ottawa, Les Presses de l'Université d'Ottawa.

BEYER, Stephen, et Axel KAEHNE (2008). «The transition of young people with learning disabilities to employment: What works? ", Journal on Developmental Disabilities, Vol. 14, № 1, p. 85-94.

BOUCK, Emily C. (2004). «State of curriculum for secondary students with mild mental retardation », Education and Training in Developmental Disabilities, Vol. 39, № 2, p. 169-176.

BURCHARDT, Tania, Julian LE GRAND et David PIACHAUD (2002). « Degrees of Exclusion: Developing a Dynamic Multidimensional Measure », dans John Hills, Julian Le Grand et David Piachaud (dirs.), Understanding Social Exclusion, Oxford, Oxford University Press.

CARDINAL, Linda, Natasha PLOURDE et Virginie COBIGO (2017). La francophonie ontarienne et les politiques gouvernementales dans le domaine de la déficience intellectuelle : un état des lieux, Université d'Ottawa, Groupe de recherche sur l'inclusion sociale.

CARROLL, Stuart Z., E. Richard BLUMBERG et Jerry G. PETROFF (2008). « The promise of liberal learning: creating a challenging postsecondary curriculum for youth with intellectual disabilities ", Focus on Exceptional Children, Vol. 40, № 9, p. 1-12.

COBIGO, Virginie, et collab. (2016). «Social inclusion: A proposed framework to inform policy and service outcomes evaluation ", Inclusion, Vol. 4, p. 226-238, DOI : 10.1352/2326-6988-4.4.226.

COBIGO, Virginie, et collab. (2012). Shifting our conceptualization of social inclusion, « Stigma Research and Action », Vol. 2, No 2, p. 75-84.

COLLĖGES DE L'ONTARIO (2017). Programmes d'éducation inclusive des collèges de l'Ontario, réf. du 5 octobre 2017, http://www.collegesdelontario.ca/ResultatRecherche/\%C3\%89DUCATIONET-SERVICES-COMMUNAUTAIRES-ET-SOCIAUX-INCLUSION-SCOLAIRE/_N-lqjt

COORDINATION DES SERVICES POUR LES PERSONNES AYANT UNE DÉFICIENCE INTELLECTUELLE (2017). «À propos de nous », réf. du 11 octobre 2017, https://scsottawa.on.ca/fr/about-us/

CULNANE, Mary, Laura T. EISENMAN et Aideen MURPHY (2016). « College peer mentoring and students with intellectual disability: Mentors' perspectives on relationship dynamics ", Inclusion, Vol. 4, No 4, p. 257-269.

DOLYNIUK, Chrystina A., et collab. (2002). «Students with developmental disabilities go to college: description of a collaborative transition project on a regular college campus ", Focus on Autism and Other Developmental Disabilities, Vol. 17, № 4, p. 236-241. 
DUMAIS, Lucie, Alexandra PROHET et Léonie ARCHAMBAULT (2012). "Insertion en emploi et TED : analyse des retombées et des enjeux politiques d'un projet pilote ", Revue francophone de la déficience intellectuelle, Vol. 23, p. 128-142, DOI : 10.7202/1015668ar.

FERRARI, Lea, Laura NOTA et Salvatore SORESI (2008). "Conceptions of work in Italian adults with intellectual disability ", Journal of Career Development, Vol. 34, № 4, p. 438-464, DOI : $10.1177 / 0894845308316295$.

GIUST, Amanda M., et Diana M. VALLE-RIESTRA (2017). "Supporting mentors working with students with intellectual disabilities in higher education ", Journal of Intellectual Disabilities, Vol. 21, № 2, p. 144-157, DOI : 10.1177/1744629516651019.

GOUVERNEMENT DU CANADA (2017). Fonds d'intégration pour les personnes handicapées, réf. du 15 septembre 2017, https:/www.canada.ca/fr/emploi-developpement-social/services/financement/ fonds_integration/guide-application.html

GOUVERNEMENT DU CANADA (2017). Loi sur l'équité en matière d'emploi, réf. du 15 septembre 2017, http://laws.justice.gc.ca/fra/lois/e-5.401/page-1.html

GOUVERNEMENT DE L'ONTARIO (2017). "À propos des lois sur l'accessibilité », réf. du 15 septembre 2017, https://www.ontario.ca/fr/page/propos-des-lois-sur-laccessibilite

GRIGAL, Meg, Debra HART et Alberto MIGLIORE (2011). «Comparing the transition planning, postsecondary education, and employment outcomes of student with intellectual and other disabilities ", Career Development for Exceptional Individuals, Vol. 34, No 1, p. 4-17, DOI : $10.1177 / 0885728811399091$.

HALL, Alison Cohen, et John KRAMER (2009). "Social capital through workplace connections: opportunities for workers with intellectual disabilities ", Journal of Social Work and Disability Rehabilitation, Vol. 8, p. 146-170.

HALL, Maeda, Harold L. KLEINERT et Jacqueline Farmer KEARNS (2000). "Going to College! Postsecondary Programs for Students with Moderate and Severe Disabilities ", TEACHING Exceptional Children, Vol. 32, № 3, p. 58-65.

HALL, Sarah A. (2009). "The social inclusion of people with disabilities: A qualitative meta-analysis ", Journal of Ethnographic and Qualitative Research, Vol. 3, p. 162-173.

HART BARNETT, Juliet E., et Robert CRIPPEN (2014). "Eight Steps to School-Based Employment Training for Adolescents with Autism Spectrum Disorder and Intellectual Disability ", Physical Disabilities: Education and Related Services, Vol. 33, No 2, p. 1-15, DOI : $10.14434 /$ pders.v33i2.5186.

HAUTE AUTORITÉ DE SANTÉ (2016). Guide méthodologique : Élaboration de recommandations de bonne pratique. Méthode "Recommandations pour la pratique clinique », réf. du 15 septembre 2017, https://www.has-sante.fr/portail/upload/docs/application/pdf/2011-01/guide_methodologique_ recommandations_pour_la_pratique_clinique.pdf 
HAUTE AUTORITÉ DE SANTÉ (2013). État des lieux : Niveau de preuve et gradation des recommandations de bonne pratique, réf. du 15 septembre 2017, https://www.has-sante.fr/portail/ upload/docs/application/pdf/2013-06/etat_des_lieux_niveau_preuve_gradation.pdf

INSTITUTE FOR RESEARCH AND DEVELOPMENT ON INCLUSION AND SOCIETY (2014). Employment of People with Developmental Disabilities in Canada: Six Key Elements for an Inclusive Labour Market, réf. du 11 octobre 2017, https://irisinstitute.files.wordpress. com/2012/01/six-key-elements-for-an-inclusive-labour-market.pdf

JONES, Melissa M., et Zach GOBLE (2012). «Creating effective mentoring partnerships for students with intellectual disabilities on campus ", Journal of Policy and Practice in Intellectual Disabilities, Vol. 9, № 4, p. 270-278, DOI : 10.1111/jppi.12010.

JUDGE, Sharon, et Dolores Izuzquiza GASSET (2015). « Practice Brief: Inclusion in the Workforce for Students with Intellectual Disabilities: A Case Study of a Spanish Postsecondary Education Program ", Journal of Postsecondary Education and Disability, Vol. 28, № 1, p. 121-127.

KAEHNE, Axel, et Stephen BEYER (2013). "Supported employment for young people with intellectual disabilities facilitated through peer support: A pilot study ", Journal of Intellectual Disabilities, Vol. 17, No 3, p. 236-251, DOI : 10.1177/1744629513495265.

KLEINERT, Harold L., et collab. (2012). «Students with Intellectual Disabilities Going to College? Absolutely! », TEACHING Exceptional Children, Vol. 44, № 5, p. 26-35.

LIN, Elizabeth, et collab. (2013). " Using administrative health data to identify individuals with developmental disabilities: A comparison of algorithms ", Journal of Intellectual Disability Research, Vol. 57, № 5, p. 462-477.

LINDSAY, Sally, Laura R. HARTMAN et Melissa FELLIN (2016). «A systematic review of mentorship programs to facilitate transition to post-secondary education and employment for youth and young adults with disabilities ", Disability and Rehabilitation, Vol. 38, No 14, p. 1329-1349, DOI : $10.3109 / 09638288.2015 .1092174$.

LIRETTE, Sylvie, et collab. (2015). " Handicap et monde du travail : pratiques et perceptions ", Reflets : revue d'intervention sociale et communautaire, Vol. 21, $\mathrm{N}^{\circ} 2$, p. 131-160, URI : http://id.erudit.org/iderudit/1035436ar, DOI : 10.7202/1035436ar.

LLYOD, Chris, Samson TSE et Frank P. DEANE (2006). « Community participation and social inclusion: How practitioners can make a difference ", Australian e-Journal for the Advancement of Mental Health, Vol. 5, № 3, p. 185-194.

LYSAGHT, Rosemary, et collab. (2017). «Inclusion through work and productivity for persons with intellectual and developmental disabilities ", Journal of Applied Research in Intellectual Disabilities, DOI : 10.1111/jar.12284, [diffusion en ligne avant l'impression : Epub 2016 Août 18]. 
LYSAGHT, Rosemary, et collab. (2014). "Work preparation and participation in Ontario for persons with intellectual and developmental disabilities - A cross-region analysis ", Journal on Developmental Disabilities, Vol. 20, № 2, p. 34-43.

LYSAGHT, Rosemary, Hélène OUELLETTE-KUNTZ et Cheng-Jung LIN (2012). " Untapped potential: Perspectives on the employment of people with intellectual disability ", Work, Vol. 41, No 4, p. 409-422.

LYSAGHT, Rosemary, Hélène OUELLETTE-KUNTZ et Carole MORRISON (2009). " Meaning and value of productivity to adults with intellectual disabilities ", Intellectual and Developmental Disabilities, Vol. 47, № 6, p. 413-424, DOI : 10.1352/1934-9556-47.6.413.

LYSAGHT, Rosemary, Virginie COBIGO et Kate HAMILTON (2012). «Inclusion as a focus of employment-related research in intellectual disabilities from 2000 to 2010: a scoping review ", Disability and Rehabilitation, Vol. 34, № 16, p. 1339-1350.

MARTIN, Lynn, et Virginie COBIGO (2011). « Definitions matter in understanding social inclusion ", Journal of Policy and Practice in Intellectual Disabilities, Vol. 8, No 4, p. 276-282.

MAUTZ, Denise, Keith STOREY et Nick CERTO (2001). « Increasing integrated workplace social interactions: the effects of job modification, natural supports, adaptive communication instruction, and job coach training ", The Journal of the Association for Persons with Severe Handicaps, Vol. 26, № 4, p. 257-269.

MELNYK, Bernadette Mazurek, et Ellen FINEOUT-OVERHOLT (2011). Evidence-Based Practice in Nursing and Healthcare: A Guide to Best Practice, Philadelphia, Lippincott, Williams \& Wilkins.

MINISTĖRE DES SERVICES SOCIAUX ET COMMUNAUTAIRES (2017). « Au sujet de l'aide sociale en Ontario ", réf. du 15 septembre 2017, http://www.mcss.gov.on.ca/fr/mcss/programs/social/index.aspx

MINISTĖRE DES SERVICES SOCIAUX ET COMMUNAUTAIRES (2017). "Services aux personnes ayant une déficience intellectuelle en Ontario ", réf. du 15 septembre 2017, http://www.mcss.gov.on.ca/fr/mcss/programs/developmental/index.aspx

MINISTÈRE DES SERVICES SOCIAUX ET COMMUNAUTAIRES (2017). « Loi », réf. du 15 septembre 2017, http://www.mcss.gov.on.ca/fr/mcss/programs/developmental/improving/ legislation.aspx

MINISTĖRE DES SERVICES SOCIAUX ET COMMUNAUTAIRES (2014). Programme Passeport: Lignes directrices du programme Passeport s'adressant aux adultes ayant une déficience intellectuelle et à leurs fournisseurs de soins, réf. du 4 octobre 2017, http://www.mcss.gov.on.ca/documents/fr/ $\mathrm{mcss} /$ publications/developmental/passport/Passport_Program_Guidelines-fr.pdf

PATTON, James R., et collab. (1996). «Individuals with Mental Retardation: Postsecondary Outcomes and Implications for Educational Policy ", Education and Training in Mental Retardation and Developmental Disabilities, Vol. 31, No 2, p. 75-85. 
POTVIN, Lynne A., Linda CARDINAL et Virginie COBIGO (2017). Recension des écrits scientifiques sur la formation professionnelle et le soutien à l'emploi des personnes ayant une défcience intellectuelle, Ottawa, Université d'Ottawa, Groupe de recherche sur l'inclusion sociale en Ontario, ISBN : 978-2-9817161-0-1.

RANDT, Nicole (2011). « Social Inclusion for People with Intellectual Disabilities », Emerging Practice CATs, Paper 6, réf. du 15 septembre 2017, http://commons.pacificu.edu/cgi/viewcontent.cgi?article=1006\&context=emerge

REGROUPEMENT DES PARTENAIRES FRANCOPHONES (2017a). «Déficience intellectuelle», réf. du 11 octobre 2017 ,

http://regroupementdespartenaires.ca/index.cfm?Voir=sections\&Id=1097\&M=367\&Repertoi re_No=2137984665

REGROUPEMENT DES PARTENAIRES FRANCOPHONES (2017b). «Qui nous sommes », réf. du 11 octobre 2017, http://regroupementdespartenaires.ca/index.cfm?Voir=menu\&Repertoire $\mathrm{No}=2137984665 \& \mathrm{M}=3503$

SERVICES DE L'ONTARIO POUR LES PERSONNES AYANT UNE DÉFICIENCE INTELLECTUELLE (2017). Réf. du 15 novembre 2017, https://www.sopdi.ca/

STATISTIQUE CANADA (2006). Participation and Activity Limitation Survey 2006: Tables, Ottawa, Minister of Industry.

THOMA, Colleen A., et collab. (2011). «Participation in Postsecondary Education for Students with Intellectual Disabilities: A Review of the Literature 2001-2010 ", Journal of Postsecondary Education and Disability, Vol. 24, № 3, p. 175-191.

TURCOTTE, Martin (2014). "Persons with disabilities and employment ", Insights on Canadian Society, Statistics Canada Catalogue no. 75-006-X, réf. du 11 octobre 2017, http://www.statcan.gc.ca/pub/75-006-x/2014001/article/14115-eng.htm

UNITED NATIONS (2006). Convention on the Rights of Persons with Disabilities (CRPD), Geneva, Switzerland: United Nations.

VILÀ, Montserrat, Maria PALLISERA et Judit FULLANA (2007). « Work integration of people with disabilities in the regular labour market: What can we do to improve these processes? ", Journal of Intellectual and Developmental Disability, Vol. 32, № 1, p. 10-18.

WEIR, Cate (2004). « Person-Centered and Collaborative Supports for College Success », Education and Training in Developmental Disabilities, Vol. 39, № 1, p. 67-73. 